[Chem. Pharm. Bull.

35( 7 )2949-2957(1987)

\title{
Coating of Pharmaceutical Powders by Fluidized Bed Process. I. Aqueous Enteric Coating with Methacrylic Acid-Ethylacrylate Copolymer and the Dissolution Behavior of Products
}

\author{
Yoshinobu FuKUmori, ${ }^{*},{ }^{a}$ Tomoaki FuKUda, ${ }^{a}$ Yoshinobu Hanyu, ${ }^{a}$ \\ YOSHIKAZU TAKEUCHI ${ }^{a}$ and YoshIFUMI OSAKO ${ }^{b}$ \\ Faculty of Pharmaceutical Sciences, Kobe-Gakuin University, ${ }^{a}$ \\ Arise, Igawadani-cho, Nishi-ku, Kobe 673, Japan and Fuji \\ Paudal, ${ }^{b}$ Chuo 2-2-30, Jyoto-ku, Osaka 536, Japan
}

(Received January 5, 1987)

\begin{abstract}
Phenacetin and lactose powders, as models of slightly and highly water-soluble drugs, respectively, were coated by means of the Wurster process.

Phenacetin ( $80-250$ mesh) was a fragile crystalline material and hence fluidization had to be moderate. As a result, the agglomeration of some particles or the adhesion of small particles to large particles could not be avoided. However, the particles coated with triacetin(plasticizer)-containing lacquer $(60 \%$ as dry lacquer relative to the core material) released only $6.0 \%$ of phenacetin in JP XI disintegration medium No. 1. This result suggested that slight agglomeration did not prevent the encapsulation. When a polyethyleneglycol 6000 and polysorbate 80 mixture was used as a plasticizer in the coating of phenacetin, the membrane became more permeable than when triacetin was used.

With lactose ( $100 \mathrm{mesh}$ ), which is a comparatively hard crystalline material, discretely encapsulated particles could easily be produced. The lactose particles coated with triacetin- and with polyethyleneglycol 6000-polysorbate 80 -containing lacquer exhibited identical dissolution properties. Their dissolution profiles were characterized by a lag time, which increased with the amount of coating applied, followed by a rapid release. This lag time was the period needed for lactose crystals to be dissolved in rapidly taken up water. The inward flow of water should prevent lactose from diffusing out during the lag time. The high solubility and high permeation rate of lactose and the large specific surface area of particles produced by the Wurster process presumably account for the rapid release after the lag time. These dissolution profiles of coated lactose particles suggested that they should be useful as a model to study oral drug delivery systems with various lag times of dissolution.
\end{abstract}

Keywords__enteric coating; powder; fluidized bed; dissolution; lactose; phenacetin; microencapsulation; Wurster process; methacrylic acid-ethylacrylate copolymer

The fluidized bed coating process is already a widely used technique for tablets and granules which have a large particle size. Recently, dosage forms composed of coated small particles have been proposed to be useful because of their gastric emptying characteristics, rapid response to the medium and so on. ${ }^{1)}$ However, small particles sprayed in the conventional fluidized bed tend to be agglomerated. Thus, the Wurster process has been proposed for powder coating, ${ }^{2)}$ since the rapidly recycled particle flow is expected to prevent agglomeration as a result of the high-speed particle motion. However, it is still not clear what conditions of spraying and fluidizing and what properties of powders result in the particles with desired properties.

A serious problem in powder coating is that a large amount of coating material may be needed to encapsulate fine powders with a large specific surface area, whereas $20-30 \%$ coating is usually sufficient for tablets and granules. This may make the practical application 
of powder coating too time-consuming and expensive.

Preliminary experiments to obtain some fundamental data on the powder coating were planned. An aqueous enteric coating was applied by the Wurster process with a methacrylic acid-ethylacrylate $(1: 1)$ copolymer dispersion, a typical aqueous coating material, and the dissolution behaviour of the products was evaluated in an aqueous acidic medium.

\section{Experimental}

Materials_-As core materials, lactose (DMV 100 M) and phenacetin (JPX grade, Hoei Yakko) were used. They were regarded as models of hydrophilic and hydrophobic drugs, respectively. Purchased phenacetin powder with a large particle size was forced through an 80 mesh sieve and particles under 250 mesh were removed by the use of an air-jet sieve (Alpine). An aqueous dispersion (30\% dry lacquer) of methacrylic acid-ethylacrylate copolymer, (MA-EA, Eudragit L30D-55, Röhm Pharma), was used as the coating material, and triacetin (TA) or polyethyleneglycol 6000 -polysorbate 80 (PEG-PS, Nakarai Chemicals) was used as the plasticizer. Talc (JP XI grade, Maruishi) was used as purchased.

Coating Apparatus - A Glatt GPCG-1 apparatus was used.

Dissolution-Dissolution tests were performed on an NTR 5S3 dissolution apparatus (Toyama Sangyo) by the JP XI paddle method at $200 \mathrm{rpm}$ and $37^{\circ} \mathrm{C}$. The dissolution medium was JP XI disintegration medium No. 1 (pH 1.2). The sample weight was $150 \mathrm{mg}$ as core material. An aliquot of $1 \mathrm{ml}$ was tken through a $0.45 \mu \mathrm{m}$ filter (Ekicrodisc 3 , Gelman Sciences Japan, Ltd.) every $30 \mathrm{~min}$. The phenacetin concentration in the sample was determined by measuring the absorbance at $245 \mathrm{~nm}$ on a Shimazu UV 190 spectrophotometer. The lactose concentration was detected by the phenol-sulfuric acid method.

Sieve Analysis - Particle size distribution was determined by sieve analysis with a row-tap shaker (Iida Seisakusho Co., Ltd.). The shaking time was $10 \mathrm{~min}$ and the charged weight was $100 \mathrm{~g}$.

Scanning Electron Microscopy (SEM) — _SEM was performed on Hitachi S430.

Polarizing Microscopy_An Olympus POM polarizing microscope was used with a heating stage (MHS, Union Optical).

\section{Results}

\section{Coating of Phenacetin and Its Dissolution}

Phenacetin crystals were used as a model of a fragile core material, ${ }^{3)}$ and as a model hydrophobic drug. The operating conditions and the composition of the spray dispersion are shown in Tables I and II, respectively. Until the coating reached $20 \%$ as MA-EA dry lacquer, the fluidizing conditions had to be moderate in order to avoid fracture of the crystals. During that stage, the powder was, therefore, under conditions where agglomeration could easily occur. To avoid agglomeration, talc was conventionally added to the spray dispersion (Table II) and, in addition, 70 or $150 \mathrm{~g}$ of talc powder was gradually inserted into the coating chamber in coating with TA or with PEG-PS, respectively. The larger amount of talc added in

Table I. Operating Conditions in Coating Phenacetin. Powder

\begin{tabular}{|c|c|c|c|}
\hline Core material & & Phenacetin & \\
\hline Mesh size & & $80-250$ & \\
\hline Charged weight $(\mathrm{g})$ & & 500 & \\
\hline Plasticizer & TA & & PEG-PS \\
\hline Inlet air temperature $\left({ }^{\circ} \mathrm{C}\right)$ & \multicolumn{3}{|c|}{60} \\
\hline Material temperature $\left({ }^{\circ} \mathrm{C}\right)$ & $33 \rightarrow 36$ & & $33 \rightarrow 36$ \\
\hline Outlet air temperature $\left({ }^{\circ} \mathrm{C}\right)$ & $31 \rightarrow 32$ & & $31 \rightarrow 33$ \\
\hline Air flow rate $\left(\mathrm{m}^{3} / \mathrm{min}\right)$ & $0.75 \rightarrow 0.9$ & & $0.75 \rightarrow 1.25$ \\
\hline Spray rate $(\mathrm{ml} / \mathrm{min})$ & \multicolumn{3}{|c|}{6.9} \\
\hline Spray pressure (atm) & $1.5 \rightarrow 2.0$ & & $1.5 \rightarrow 2.6$ \\
\hline Diameter of spray nozzle $(\mathrm{mm})$ & \multicolumn{3}{|c|}{0.8} \\
\hline Drying conditions & \multicolumn{3}{|c|}{$60^{\circ} \mathrm{C}, 60 \mathrm{~min}$} \\
\hline
\end{tabular}


TABLE II. Composition of Spray Dispersion

\begin{tabular}{lccc}
\hline \hline MA-EA dispersion & $1000 \mathrm{~g}$ & & $1000 \mathrm{~g}$ \\
TA & $30 \mathrm{~g}$ & PEG 6000 & $30 \mathrm{~g}$ \\
& & Polysorbate 80 & $15 \mathrm{~g}$ \\
Talc & $90 \mathrm{~g}$ & & $90 \mathrm{~g}$ \\
Water & Added & & Added \\
Total & $2400 \mathrm{ml}$ & & $2400 \mathrm{ml}$ \\
Total solid & $420 \mathrm{~g}(84 \%)$ & & $435 \mathrm{~g}(87 \%)$ \\
MA-EA dry lacquer & $60 \%$ & $60 \%$ \\
\hline
\end{tabular}

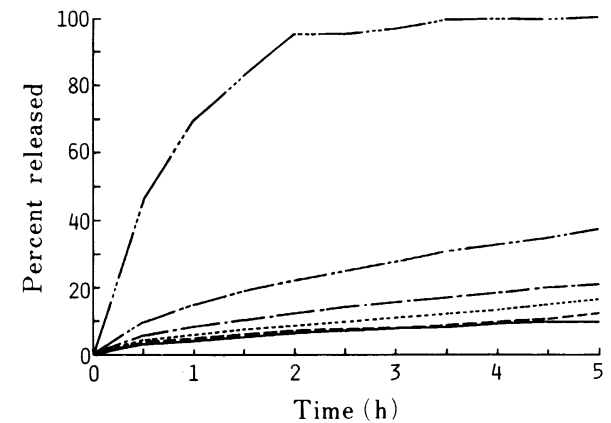

Fig. 1. Dissolution of Phenacetin Coated with TA-Containing Lacquer in JPXI Disintegration Medium No. 1 ( $\mathrm{pH}$ 1.2)

MA-EA dry lacquer relative to core material $(\%)$ - -.- $-10 ;-\cdots, 20 ;--\ldots, 30 ;----, 40 ;----$ $50 ;-60$.

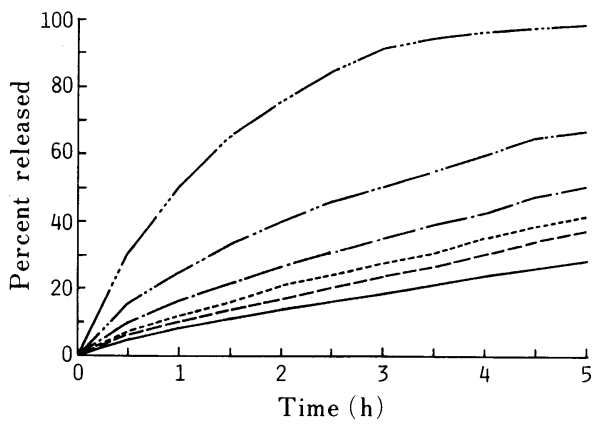

Fig. 2. Dissolution of Phenacetin Coated with PEG-PS Containing Lacquer in JPXI Disintegration Medium No. 1 ( $\mathrm{pH}$ 1.2)

MA-EA dry lacquer relative to core material $(\%)$ --- - , 10; - --, 20 ; -- - 30 ; -----, 40; ----, $50 ;-60$.

coating with PEG-PS was due to the more wettable character of the sprayed powder. At $20 \%$ coating, where the coated particles seemed not to be fractured any more, the conditions were altered as shown by the arrows in Table I.

In this study, the inlet air temperature was kept at $60^{\circ} \mathrm{C}$. The inlet air rate and the spray pressure were set so that particles would not be fractured. The spray rate was also adjusted so that the air-suspended particles could be slightly wetted in the upper part of chamber and the fluidized bed at the bottom could be dry.

The dissolution curves for the product coated with TA are shown in Fig. 1. The coating with $20 \%$ MA-EA dry lacquer relative to phenacetin provided a fairly good barrier against phenacetin release. The release at $2 \mathrm{~h}$ was $6.0 \%$ at $60 \%$ coating. This approaches a practical level for enteric coating. Figure 1 also suggests that sustained-release products may be produced by less than $20 \%$ coating.

The dissolution curves for the product coated with PEG-PS are shown in Fig. 2. The membrane with PEG-PS was a less effective barrier against phenacetin diffusion than the membrane containing TA. As a result, a larger amount of coating material would be needed for enteric coating.

\section{Coating of Lactose and Its Dissolution}

Lactose was used as a model water-soluble, hydrophilic drug. The lactose crystal is comparatively hard and can not be fractured easily. ${ }^{3)}$ Hence, lactose powder could be fluidized under more severe conditions (Table III) than phenacetin (Table I), so that the particles were not agglomerated.

The dissolution curves for the lactose powder coated with TA are shown in Fig. 3. When 
TABLE III. Operating Conditions in Coating Lactose Powder

\begin{tabular}{lc}
\hline \hline Core material & Lactose \\
Mesh size & 100 \\
Charged weight $(\mathrm{g})$ & 500 \\
Plasticizer & TA or PEG-PS \\
Inlet air temperature $\left({ }^{\circ} \mathrm{C}\right)$ & 60 \\
Material temperature $\left({ }^{\circ} \mathrm{C}\right)$ & 40 \\
Outlet air temperature $\left({ }^{\circ} \mathrm{C}\right)$ & 35 \\
Air flow rate $\left(\mathrm{m}^{3} / \mathrm{min}\right)$ & 1.7 \\
Spray rate $(\mathrm{ml} / \mathrm{min})$ & 6.4 \\
Spray pressure $($ atm $)$ & 2.5 \\
Diameter of spray nozzle $(\mathrm{mm})$ & 0.8 \\
Drying conditions & $60{ }^{\circ} \mathrm{C}, 60 \mathrm{~min}$ \\
\hline
\end{tabular}

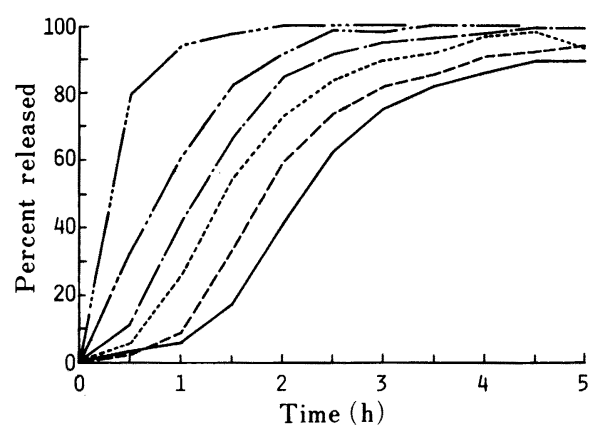

Fig. 3. Dissolution of Lactose Coated with TAContaining Lacquer in JPXI Disintegration Medium No. 1 (pH 1.2)

TA dry lacquer relative to core material $(\%)$ : $-\cdots-, 10 ;-\cdots, 20 ;-\cdots, 30 ; \cdots,-\cdots, 40 ;-\cdots$, $50 ;-, 60$.

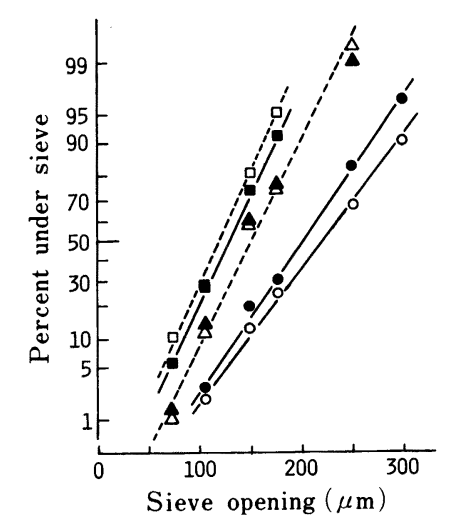

Fig. 4. Particle Size Distributions of Uncoated and Coated Powders on a Normal Probability Scale as Determined by Sieve Analysis

Symbols and $50 \%$ diameters $(\mu \mathrm{m})$ :

\begin{tabular}{|c|c|c|c|c|c|c|}
\hline \multirow{3}{*}{$\begin{array}{l}\text { Core material } \\
\text { Plasticizer }\end{array}$} & \multicolumn{2}{|c|}{ Uncoated } & \multicolumn{4}{|c|}{ Coated } \\
\hline & \multirow{2}{*}{$\begin{array}{c}\text { Lactose } \\
-\end{array}$} & \multirow{2}{*}{ Phenacetin } & \multicolumn{2}{|c|}{ Lactose } & \multicolumn{2}{|c|}{ Phenacetin } \\
\hline & & & PEG-PS & TA & PEG-PS & TA \\
\hline $50 \%$ diameter & 120 & 128 & 150 & 150 & 200 & 223 \\
\hline Symbol & $\square$ & $\mathbf{\square}$ & $\Delta$ & $\triangle$ & 0 & 0 \\
\hline
\end{tabular}

the plasticizer was changed to PEG-PS, the dissolution curves (not shown here) were almost identical with those in Fig. 3. Figure 3 shows that the dissolution of lactose had a lag time above $30 \%$ coating and became very rapid after the lag time. The rate at the rapid dissolution stage changed only moderately above $20 \%$ coating, suggesting that the particles were almost wholly encapsulated at $20 \%$ coating.

\section{Particle Size Distribution}

The particle size distributions are shown in Fig. 4 for the raw powders and the products. The coated phenacetin powder had a remarkably increased particle size (see the legend to Fig. 4). This was because phenacetin was fluidized under relatively mild conditions, and hence 

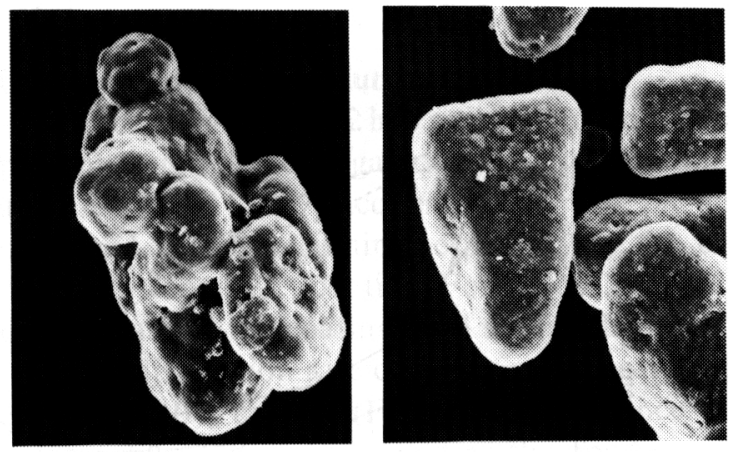

$100 \mu \mathrm{m}$ b
Fig. 5. Photographs of Coated Particles $(\times 300)$

Core material and plasticizer: a, phenacetin-TA; b, lactose-TA.

small particles could adhere to large particles and even large particles could be agglomerated to some extent. The photograph in Fig. 5a shows this feature of the particles.

On the other hand, the particle size of lactose powder was not much enlarged by coating, presumably because of the more severe fluidizing conditions used in coating lactose powder (Table III). The powder visualized by SEM consisted of discrete particles, as shown in Fig. $5 \mathrm{~b}$. The increase of the $50 \%$ diameter by $30 \mu \mathrm{m}$ was clearly due to the thickness of the formed membrane.

\section{Discussion}

\section{Particle Flow in the Wurster Chamber}

In the Wurster chamber (Fig. 6), particles are forced into a cylindrical partition by air flow and sprayed there. The particles thereafter fall with the recycling air flow in the upper part of chamber. The particles deposited outside the partition are fluidized by distributed air and again drawn into the partition. ${ }^{2)}$

To produce discretely coated particles without agglomeration, it is desirable for particles to be sprayed and dried without flocculation. Even if particles were sprayed into flocs in the partition or in the upper part of chamber, they had to be separated into discrete particles at latest until they were again forced into the partition. Although the lactose particles were kept separate under the severe fluidizing conditions, the phenacetin particles gradually agglomerated because of their more moderate motion (Tables I and III).

To obtain a homogenously coated product, it is desirable for all particles to have the same recycling time. For a powder with a wide particle size distribution, however, this is difficult in principle. In particular, small particles easily adhere to the wall surface and the filter through electrostatic attraction or moisture on the particle surfaces. In addition, small particles may sometimes be ejected to the filter. In the runs with lactose and phenacetin reported here, the small particles were carried by adhering temporarily to the slightly wet large particles.

In the case of phenacetin, which is composed of fragile crystalline particles, like many other pharmaceutical powders, relatively moderate conditions-were employed at the initial stage of coating to avoid fracture. Sufficiently coated crystals are, however, not so easily fractured as the uncoated crystals, and thus conditions were made more severe after the initial stage to avoid agglomeration of the coated particles.

Talc powder added to avoid excessive agglomeration at the initial stage of phenacetin coating could also prevent the adhesion of small particles to the wall, especially to the upper part of the chamber. Since the talc powder adhering to phenacetin particles acted as an anti- 


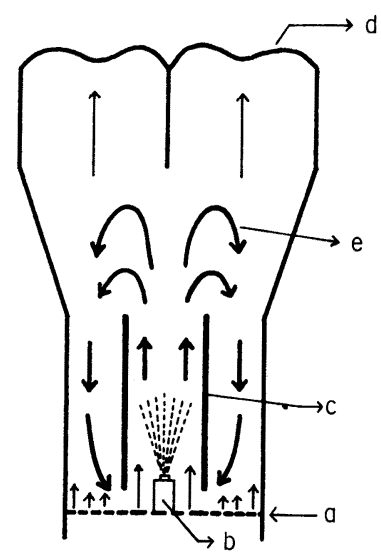

Fig. 6. Schematic Diagram of the Wurster Coating Chamber

a, air distributor; b, spray; c, cylindrical partition; $\mathrm{d}$, bag filter; e, particle and air flow.

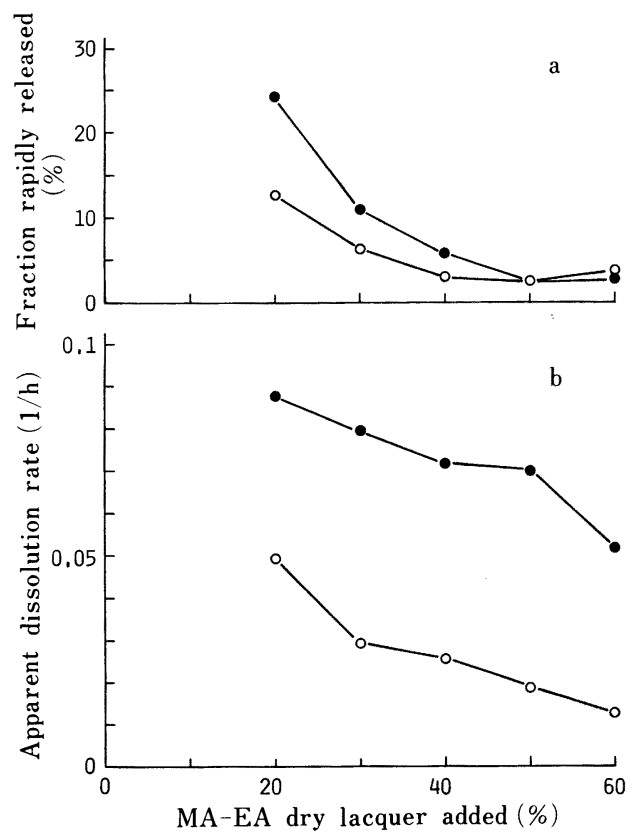

Fig. 8. Characteristic Parameters of Dissolution of Phenacetin

a. Fraction of particles which exhibited rapid dissolution. b. Apparent dissolution rate. Plasticizer used in coating: $\mathrm{O}$, TA;, PEG-PS.

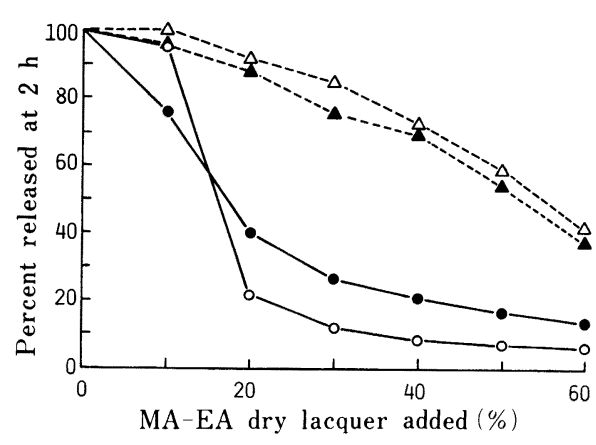

Fig. 7. Release after $2 \mathrm{~h}$ from Coated Particles in JP XI Disintegration Medium No. 1 (pH 1.2)

Core material and plasticizer: $\bigcirc$, phenacetin-TA; -, phenacetin-PEG-PS; $\triangle$, lactose-TA; $\boldsymbol{\Delta}$, lactosePEG-PS.

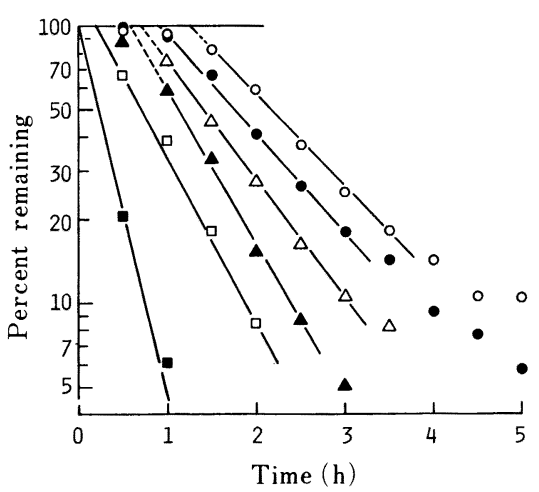

Fig. 9. Semi-log Plots of Percent of Lactose Remaining

MA-EA dry lacquer relative to core material $(\%)$ :

$\mathbf{\square}, 10 ; \square, 20 ; \mathbf{\Delta}, 30 ; \triangle, 40 ; \boldsymbol{\bullet}, 50 ; \bigcirc, 60$.

agglomerant, the coating could be performed under wet conditions. As a result, the temporary capture of small particles by wet large particles was accelerated and effectively prevented particle adhesion to the wall. Since particle adhesion to the wall would result in insufficiently coated particles, the addition of talc was critical for successful coating of phenacetin. Because excessive talc addition might prevent a compact membrane being formed, it was limited to 
$20 \%$ coating. This approach should be generally useful for coating of fragile particles.

\section{Characteristics of Dissolution}

Since a lag time of $2 \mathrm{~h}$ is required in drug release from enteric-coated pharmaceuticals to prevent drug release in gastric juice, the amount of drug released in $2 \mathrm{~h}$ is plotted in Fig. 7 against MA-EA dry lacquer added. For phenacetin, the amount released in $2 \mathrm{~h}$ rapidly decreased at the beginning, especially in the case of TA. However, the change becomes moderate above $20 \%$ coating. For lactose, neither of the membranes sufficiently restrained the drug release at the coating level studied here.

The dissolution rate of phenacetin became almost constant after the initial rapid release except for $10 \%$ coating (Figs. 1 and 2). Hence, the slope of the linear regression line for the data at $2.5-5 \mathrm{~h}$ was regarded as the apparent dissolution rate. The intercept of the linear portion on the ordinate could be regarded as representing the fraction of insufficiently coated particles. The results are plotted against MA-EA dry lacquer added (Fig. 8).

For phenacetin, the particles coated with PEG-PS have higher permeability above $20 \%$ coating than those coated with TA (Fig. 8). Figure 8 also shows that the fraction of initial rapid release is nearly $50 \%$ of total $2 \mathrm{~h}$ release. These results suggest that for a moderately soluble material such as phenacetin, an impermeable, homogeneously coated membrane has to be produced.

For lactose, the lag time and the rate at the stage of rapid release were characteristic parameters. Semi-log plots of the percent remaining $(R)$ are shown in Fig. 9. The slope and the intercept with $R=100(\%)$ of the linear regions of the plots gave the desired parameters. The results are plotted in Fig. 10 against MA-EA dry lacquer added.

For lactose, a water-soluble drug model, the dissolution rate was high even at $60 \%$ coating (Fig. 10). The MA-EA membrane does not seem to provide a simple diffusion barrier which is sufficient to act as an enteric coating for water-soluble core materials. On the other hand, the increase in lag time is nearly linear above $10 \%$. This suggests that the prolongation
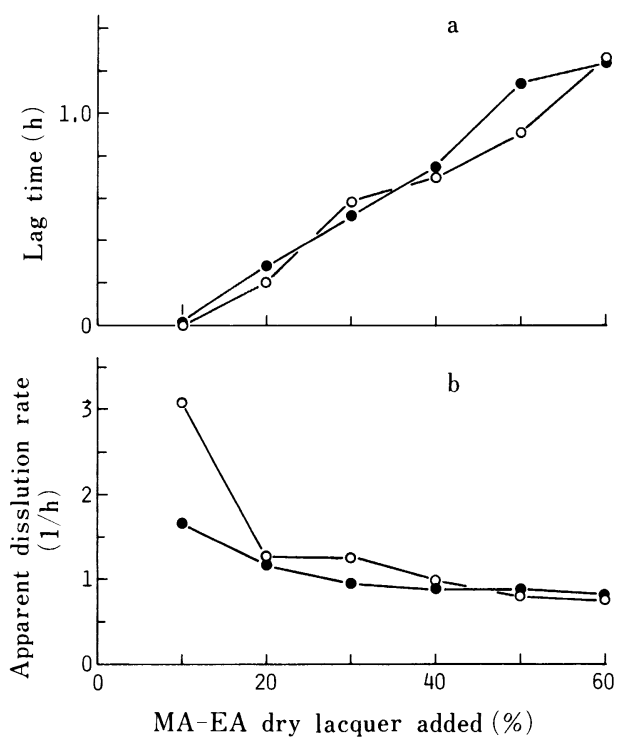

Fig. 10. Characteristic Parameters of Dissolution of Lactose

a. Lag time. b. Apparent dissolution rate. Plasticizer used in coating: $\bigcirc$, TA; 9 , PEG-PS.

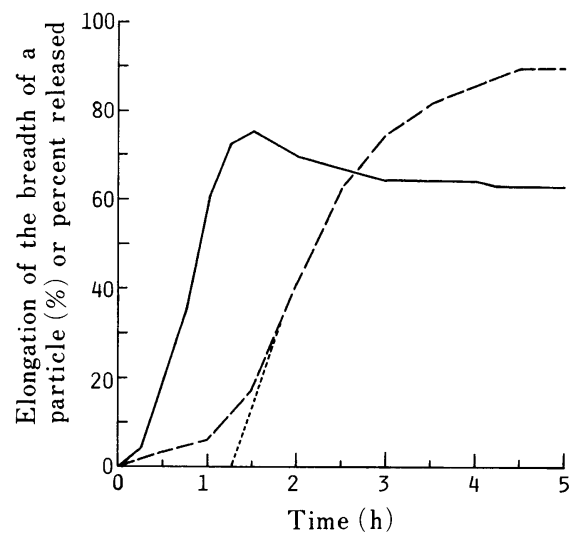

Fig. 11. Expansion of Lactose Particle $60 \%$ Coated with TA-Containing Lacquer in JP XI Disintegration Medium No. $1(\mathrm{pH} 1.2)$ at $37^{\circ} \mathrm{C}$ and the Corresponding Dissolution Curve 
of lag time is desirable for enteric coating of water-soluble drugs.

\section{Dissolution of Water-Soluble Drug from Capsules}

In this study, the dissolution profiles of lactose and phenacetin used as core materials were very different, even though the same coating material was used (Figs. 1 and 3). The main difference was in the lag time of dissolution in the case of lactose. It seemed to result only from the large difference in solubility. However, Koida et al. ${ }^{4}$ reported that ethylcellulose microcapsules of core materials with various solubilities comparable to that of lactose exhibited no lag time in the dissolution.

The membrane on lactose particles did not seem to be ruptured by stirring during the dissolution tests, when particles were filtered off and observed under an optical microscope. This indicates that the rapid dissolution after the lag time did not result from macroscopic rupture of the membrane.

When coated particles on the heated stage of a polarizing microscope were immersed in acidic medium and observed at $37^{\circ} \mathrm{C}$, the lactose particles exhibited a marked increase in their breadth, whereas only a slight increase (less than $10 \%$ ) of their length was observed. An example of such an expansion is shown in Fig. 11 with the corresponding dissolution curve. At the maximum of expansion, which coincided well with the lag time of dissolution, the dissolving core crystals just disappeared. These results suggested that the lag time in the dissolution of lactose was due to the rapid water intake, resulting from the high solubility of lactose, and the restraint of lactose diffusion by this inward water flow.

Kawashima et al. ${ }^{5}$ reported that encapsulated theophylline particles showed a lag time in dissolution. Their dissolution profiles showed less restraint at the beginning of dissolution and thereafter a slower rate of release, compared to the case of lactose reported here. The lag time in their dissolution profiles seemed to result from the swelling of the thick membrane, different from the mechanism of the time lag of dissolution reported here.

The dissolution profiles of coated lactose suggested that hydrophilic drugs coated as fine capsules by the Wurster method and orally administered may be delivered to any desired position in the gastro-intestinal tract and rapidly released there, if a $\mathrm{pH}$-independently insolule membrane with the same water- and drug-permeation characteristics as MA-EA membrane is used. The averaging effect in gastro-intestinal transit of such small particles should provide good availability of drugs delivered in this way. The present model system should be useful in providing a basis for the design of membranes with such release patterns.

\section{Conclusion}

The success of powder coating is limited by many kinds of powder properties such as particle density, crystal hardness, wettability, electrostatic charging, adhesion, particle size distribution and so on. However, even for such a fragile powder as phenacetin, a successful procedure could be found in this study. Talc powder prevented the adhesion of small particles to the wall and also prevented excessive agglomeration. The adhesion of small particles to large particles and the agglomeration of some large particles seemed not to present a serious problem. These results have important implications for the practical coating of various pharmaceutical powders.

Dissolution through the MA-EA membrane was markedly affected by the properties of the plasticizer and core material. The amount of coating materials and the kind of plasticizer have to be determined according to the required characteristics of products. In the enteric coating of a hydrophobic substance such as phenacetin, a membrane with low permeability has to be designed. In such a case, triacetin can be effectively used as a plasticizer. For a highly water-soluble substance such as lactose, a membrane with a large lag time has to be designed 
for enteric coating purposes. For sustained release, a $20 \%$ coating may be sufficient, though more than $60 \%$ coating seems to be needed for enteric coating at the present stage of study. The present model system should be useful in developing oral drug delivery system with a controlled lag time of dissolution.

\section{References}

1) a) H. Maekawa, Pharm. Tech. Jpn., 2, 767 (1986); b) Idem, ibid., 2, 581 (1986); c) K. Lehmann and D. Dreher, Pharm. Technol., 3, 53 (1979).

2) a) T. M. Hinkes, Abstracts of Papers, 19th Annual National Industrial Pharmaceutical Research Conference, Lake Delton, Wisconsin, June 1977; b) K. Lehmann and D. Dreher, Int. J. Pharm. Technol. Prod. Mfr., 2, 31 (1981); c) L. P. Amsel, O. N. Hinsvark, K. Rotenberg and J. L. Sheumảer, Pharm. Technol., 8, 28 (1984); d) A. M. Mehta and D. M. Jones, ibid., 9, 52 (1985); e) K. W. Olsen, Plant/Operations Progress, 4, 135 (1985); f) A. M. Mehta, M. J. Valazza and S. E. Abele, Pharm. Technol., 10, 46 (1986); g) H. S. Hall and R. E. Pondell, "Controlled Release Technologies: Methods, Theory, and Applications," Vol. II, CRC Press, Inc., Boca Raton, Florida, 1980, p. 133.

3) T. Fukuda, Y. Fukumori, S. Wada and Y. Hanyu, Chem. Pharm. Bull., 28, 393 (1980).

4) Y. Koida, M. Kobayashi and M. Samejima, Chem. Pharm. Bull., 34, 3354 (1986).

5) Y. Kawashima, T. Handa, A. Kasai, H. Takenaka and S. Y. Lin, Chem. Pharm. Bull., 33, 2469 (1985). 